\section{Five Cases of Anthracycline-Induced Cardio- myopathy Effectively Treated with Carvedilol}

\section{Summary}

Anthracycline, a widely used anti-cancer agent, can elicit irreversible cardiomyopathy called anthracycline-induced cardiomyopathy (ACM). In this report, we describe 5 cases of severe chronic heart failure due to ACM effectively treated with the beta-blocker, carvedilol. Their left ventricular function as well as cardiac symptoms were persistently improved after treatment with carvedilol, suggesting that carvedilol may be an effective therapeutic strategy for ACM as demonstrated in other forms of chronic heart failure.

Key words: anthracycline-induced cardiomyopathy, chronic heart failure, $\beta$-blocker, carvedilol

Anthracycline derivatives are highly useful anti-cancer agents in the treatment of neoplasmatic diseases such as acute leukemia, malignant lymphoma and so on. However, the fact that these agents have adverse cardiac effects remains a serious problem. A chronic form of cardiac toxicity is described as anthracycline-induced cardiomyopathy (ACM) and is characterized by irreversible and lethal left ventricular dysfunction $(1,2)$. Anthracycline derivatives gives rise to cardiomyopathy in a dose-dependent manner, and the incidence is reported to be as high as $35 \%$ in patients who received more than $550 \mathrm{mg} / \mathrm{m}^{2}$ of adriamycin. The mortality rate is reported to be as high as $20 \%$ among patients with symptomatic $\operatorname{ACM}(1,2)$. Carvedilol, a $\beta$-blocker, is widely used for chronic heart failure caused by idiopathic dilated cardiomyopathy (DCM) or ischemic cardiomyopathy. However, it is not known whether or not carvedilol is also effective for patients with ACM, a pathophysiologically different form of chronic heart failure.

We had 5 consecutive patients with newly diagnosed ACM between 1997 and 2002. All the patients had acute myelocytic leukemia and thus had received a chemotherapy regimen including an anthracycline derivative. Fortunately, complete remission of the primary disease was attained in all of them, however they next suffered from heart failure caused by ACM. The mean duration from the last administration of anthracycline to the onset of congestive heart failure was $31 \pm 22$ months. Conventional therapy such as diuretics, digitalis and angiotensin-converting enzyme (ACE) inhibitors were effective in controlling the pulmonary and systemic congestion although the left ventricular dysfunction and symptoms of chronic heart failure persisted thereafter (Table 1). The patients' mean left ventricular ejection frac- tion and end-diastolic left ventricular dimension shown by echocardiography were $36 \%$ and $59 \mathrm{~mm}$, respectively, even after the congestive state was controlled (Table 1, Fig. 1). We started treatment with carvedilol for these patients initially from a very low dose (as low as 1 or $2 \mathrm{mg} /$ day), similar to the way we do for patients with DCM. The dose was gradually increased up to 10 or $20 \mathrm{mg}$ per day in each patient within months. During the follow-up period, their symptoms, as indicated by NYHA functional class, as well as their left ventricular function were markedly improved. Their mean left ventricular ejection fraction and end-diastolic left ventricular dimension were improved to $53 \%$ and $50 \mathrm{~mm}$, respectively, after the treatment with carvedilol (Table 1, Fig. 1). In addition, it is of note that no patient needed rehospitalization because of recurrent congestive heart failure during the follow-up period. Usage of diuretics could be withdrawn in 2 patients after the carvedilol treatment.

\section{Discussion}

There is no clinical trial with a large number of patients evaluating the efficacy of $\beta$-blockers in ACM, since it may be difficult to carry out such a study for this rare form of heart failure. Fazio et al in Italy reported a case of ACM treated with carvedilol (3). Noori et al in the U.S. reported the possible benefit of $\beta$-blockers for ACM in 8 patients (4). It was also reported that carvedilol exerts protective effects against ACM in animal models. Along with these reports, our results suggest beneficial effects of carvedilol for the treatment of chronic heart failure caused by ACM.

Carvedilol is a unique $\beta$-blocker with some alphablocking effect and potent anti-oxidant effect, and it is the most widely used $\beta$-blocker for chronic heart failure. In the treatment of ACM, conventional therapy including diuretics, digitalis and ACE inhibitors may be widely used, although the responses to those drugs are not satisfactory. Interestingly, left ventricular function improved in our 3 patients who did not receive ACE inhibitors, suggesting that carvedilol itself had a central role in the process. The mechanisms by which carvedilol improved left ventricular function in our patients may include both its inhibition of the hyperactivity of the sympathetic nervous system and also its antioxidant effect. The latter may be of particular importance for ACM in which oxidative stress plays a major part in the mechanism (5).

Although this is an observation in a small number of patients, our findings are encouraging and it can be concluded that carvedilol may be useful in the treatment of chronic heart failure with ACM. This possibility may be generalized to other forms of drug-induced cardiomyopathy as well. 
Table 1. Clinical Characteristics and Echocardiographic Findings before and after Carvedilol Treatment in the Five Patients

\begin{tabular}{|c|c|c|c|c|c|c|c|c|c|c|c|c|}
\hline & \multirow{2}{*}{ Age/Sex } & \multirow{2}{*}{$\begin{array}{c}\text { Dose of } \\
\text { Carvedilol } \\
\text { (mg/day) }\end{array}$} & \multirow{2}{*}{$\begin{array}{c}\text { Duration of } \\
\text { Therapy } \\
\text { (mo) }\end{array}$} & \multicolumn{2}{|c|}{$\begin{array}{c}\text { NYHA } \\
\text { functional class }\end{array}$} & \multicolumn{2}{|c|}{ LVEF $(\%)$} & \multicolumn{2}{|c|}{ LVDd (mm) } & \multicolumn{3}{|c|}{ Other Drug Usage } \\
\hline & & & & Before & After & Before & After & Before & After & Diuretic & ACE-I & Digitalis \\
\hline Patient 1 & $43 / \mathrm{F}$ & 20 & 25 & III & II & 17 & 44 & 58 & 47 & + & - & - \\
\hline Patient 2 & $60 / \mathrm{M}$ & 20 & 18 & III & II & 25 & 40 & 63 & 60 & + & - & - \\
\hline Patient 3 & $43 / \mathrm{F}$ & 10 & 24 & III & II & 52 & 62 & 48 & 43 & Withdrawn & - & - \\
\hline Patient 4 & $55 / \mathrm{M}$ & 10 & 60 & III & II & 55 & 64 & 53 & 48 & Withdrawn & + & + \\
\hline Patient 5 & $62 / \mathrm{M}$ & 15 & 7 & III & II & 36 & 56 & 76 & 54 & + & + & - \\
\hline
\end{tabular}

LVEF: left ventricular ejection fraction, LVDd: diastolic left ventricular dimension, ACE-I: angiotensin-converting enzyme inhibitor. NYHA: New York Heart Association.

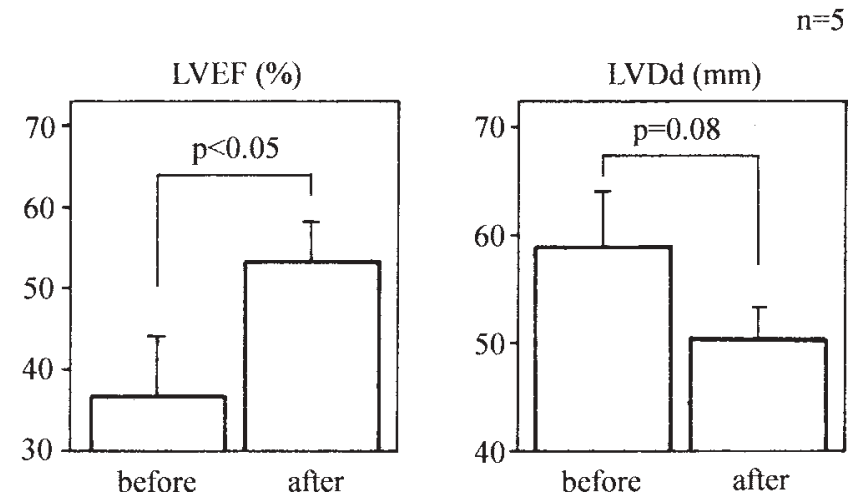

Figure 1. Left ventricular function shown by echocardiography before and after long-term treatment with carvedilol. LVEF: left ventricular ejection fraction, LVDd: diastolic left ventricular dimension. Results were expressed as mean \pm SEM. Differences in parameters before and after the treatment were compared by the paired $t$ test. A p value of $<0.05$ was considered to be statistically significant.

\author{
Yasushi MuKaI, Tomoki YoshIDA, \\ Ryuichi NaKAIKE, Naoko MuKaI, Koji IwATO*, \\ Taiichi KYO* and Shunichi KASEDA
}

From the Cardiology Division and *the Fourth Internal Medicine Division, Hiroshima Red-Cross and Atomic Bomb Survivors Hospital, Hiroshima Received for publication February 26, 2004; Accepted for publication August 10, 2004

Reprint requests should be addressed to Dr. Shunichi Kaseda, the Cardiology Division, Hiroshima Red-Cross and Atomic Bomb Survivors Hospital, 1-9-6 Sendamachi, Naka-ku, Hiroshima 730-8619

\section{References}

1) Keefe DL. Anthracycline-induced cardiomyopathy. Semin Oncol. 4 (Suppl 12): 2-7, 2001.

2) Steinherz LJ, Steinherz PG, Tan CT, Heller G, Murphy ML. Cardiac toxicity 4 to 20 years after completing anthracycline therapy. JAMA 266: 1672-1677, 1991.

3) Fazio S, Palmieri EA, Ferravante B, Bone F, Biondi B, Sacca L. Doxorubicin-induced cardiomyopathy treated with carvedilol (a case report). Clin Cardiol 21: 777-779, 1998.

4) Noori A, Lindenfeld J, Wolfel E, Ferguson D, Bristow MR, Lowes BD. Beta-blockade in adriamycin-induced cardiomyopathy. J Card Fail 6: 115-119, 2000.

5) Singal PK, Iliskovic N, Li T, Kumar D. Adriamycin cardiomyopathy: pathophysiology and prevention. FASEB J 11: 931-936, 1997. 